\title{
Research on Hot Research Topics on Personalized Learning and Its Development Trend in China:Based on Co-word Visualization Analysis from CSSCI
}

\author{
ZHOU Dongdai ${ }^{1,2,3, a *}, X$ Shuo ${ }^{1, b}$ and FAN Yaqin ${ }^{1, c}$ \\ ${ }^{1}$ School of Information and Software Engineering, Northeast Normal University, Changchun, \\ Jilin, China \\ 2Ministry of Education Digital Learning Support Engineering Technology Research Center, \\ Changchun Jilin ,China \\ 3“Internet+"Education Science and Technology Innovation Center of Jilin Province, Changchun \\ Jilin ,China \\ addzhou@nenu.edu.cn,, $439545324 @ q q . c o m,{ }^{c}$ sdnufyq@163.com
}

Keywords: Personalized learning,Co-word analysis, Visualized analysis, Development trend

\begin{abstract}
With the continuous development and change of the Times, education view is gradually shifted from teachers to students, and personalized learning begins to return to people's horizons.In this paper, based on CNKI, with co-word visualization analysis as research methods, the personalized learning research hot topic and development trend in our country are summarized and analyzed.Research shows that: the personalized learning research hot topics includes data mining, MOOC, learning environment, learning analysis, ubiquitous learning, learning style, E-learning and teaching mode and so on several aspects;Personalized learning research trend mainly involves the theoretical framework method of personalized learning, the diversification of personalized learning form, the implementation of personalized learning ways and technology and so on several directions.
\end{abstract}

\author{
我国个性化学习研究热点主题及趋势探析 \\ - - 基于 $\operatorname{CsSCl}$ 期刊的共词可视化分析 \\ 周东岱 ${ }^{1,2,3, a}$ 徐硕 ${ }^{1, \mathrm{~b}}$ 㚞雅琴 ${ }^{1,0 *}$ \\ '东北师范大学 信息与软件工程学院, 吉林长春; \\ 教育部数字化学习支撑技术工程研究中心, 吉林长春 \\ ${ }^{3}$ 吉林省 “互联网+”教育科技创新中心，吉林长春 \\ addzhou@nenu. edu. cn, ${ }^{\text {b}} 439545324 @ q q$. com, ${ }^{\text {'s }}$ dnufyq@163. com
}

关键词: 个性化学习; 共词分析;可视化分析; 发展趋势

中文摘要: 随着时代的不断发展和变革, 教育视角逐渐从教师转向学生, 个性化学习开始回 归人们的视野。本文依托 CNKI, 以共词可视化分析为研究方法, 对我国个性化学习的研究热 点主题及发展趋势进行归纳和分析。研究表明: 我国个性化学习的研究热点主题主要包括数 据挖掘、MOOC、学习环境、学习分析、泛在学习、学习风格、E-1earning 以及教学模式等几 个方面; 个性化学习研究趋势主要涉及个性化学习的理论架构方法、个性化学习的多元化存 在形式、个性化学习的实现途径与技术等几个方向。 


\section{1.引言}

个性化学习最早可追溯到我国春秋时期孔子提出“因材施教”的思想。他认为，教师应 当考虑不同学生的个体差异进行不同的教学, 主要包括学生个体的学习兴趣、学习能力等方 面。之后, 美国著名教育家杜威在其《明日之学校》一书中也曾提到 “以学生为中心” 的灵 活教育取向。以此, 个性化学习的概念一步步进入人们的视野。但是 20 世纪工业革命的发展 使得教育普及化、大班授课制等现象不断发生, 而这正是学生个性化学习的有力障碍。21 世 纪是一个充满机遇和挑战的时代, 它的到来为个体的个性化发展提供了更多的可能性。由此, 教育视角又逐渐从 “以教为主” 转向 “以学为主”。

2006 年 7 月, 在题为 “聚焦于重要之上” 的研讨会上, 新西兰教育部长史蒂夫・马哈雷 (Steve Maharey) 发表了有关个性化学习的重要演讲, 即《个性化学习: 把学生置于教育的 中心》（Personalised Learning:Putting Students at the Heart of Education），对个性化学习及其 对教育的重要影响给予了全面而深刻的阐释 ${ }^{[1]}$ 。《国家中长期教育改革和发展规划纲要

（2010-2020 年）》同样指出, 要树立多样化的人才观念, 尊重个性发展, 培养创新型和专门 化的高素质人才 ${ }^{[2]}$ 。习近平指出, 要构建网络化、数字化、个性化、终身化的教育体系, 以 推动教育变革和创新 ${ }^{[3]}$ 。

可见，个性化学习正日益受到人们的关注，逐渐回归人们的视野。但就目前的研究而言， 学者们更多的关注个性化学习的相关平台建设、资源推送、模型建构以及策略设计等相关内 容, 对其的文献综述及趋势探析等相关内容研究较少。由此, 本文以 CNKI 为依托, 以共词可 视化分析为研究方法, 对我国个性化学习的热点主题及发展趋势进行归纳分析。

\section{2. 研究设计}

\section{1 研究方法}

\section{1.共词聚类分析法}

共词分析（Co- word Analysis ${ }^{1}$ ) 法最早由法国文献计量学家提出，其思想来源于文献 计量学中引文耦合与共被引的概念, 是指以文献中词汇对或名词短语共同出现的情况为依据 来揭示学科研究中的某些现象或趋势 ${ }^{[4]}$ 。共词聚类分析法是共词分析法中较常用的一种分析 方法。它是在共词分析的基础上, 以文献中关键词共同出现的次数为研究对象, 借助聚类分 析的统计学方法, 将联系较紧密的关键词组聚类在一起, 从而简化原始关键词集中错综复杂 的关系, 以此来发现学科或主题的研究热点 ${ }^{[5]}$ 。一般来说, 关键词对在同一篇文献中出现的 次数越多，表明二者之间的关系越紧密。

2.社会网络分析法

如果将 “社会网络”比喻成一个社会群体，那么社会网络图中的一个点则代表一个行动 者, 点之间的连线代表行动者之间的联系。社会网络分析法 (Social Network Analysis Method）即是对一个社会网络群体中各行为者及其之间的关系进行量化分析的一种可视化统 计学方法 ${ }^{[6]}$ 。将其与共词分析法相结合, 并借助社会网络分析软件, 即可实现文献研究主题 的可视化。其中, 点代表研究文献中出现的关键词, 点与点之间的连线代表关键词之间的联 系。点越大, 说明该点所代表的关键词在学科研究中所占的份量越大; 连线越粗, 说明其关 键词之间的相互关系越强烈 ${ }^{[7]}$ 。通过观察分析社会网络关系图中关键词的相对位置和距离, 可以用来探究主题的结构变化和发展趋势, 并对前沿研究领域进行判断和预测 ${ }^{[8]}$ 。

\section{2 研究数据}

\section{1.数据来源}

根据布拉德福提出的文献分散规律，大量的专业文献集中在为数不多的核心期刊中 ${ }^{[9]}$ 。

1 在目前的研究文献中, 有关共词分析的英文翻译有三种形式, 即 Co-word Analysis、Co-keyword Analysis、Co-term Analysis，本文选取第一种作为 “共词分析” 的英文注释。 
由此, 本文选定教育技术学专业中具有代表性的八种 CSSCI 期刊 (包括远程教育杂志、中国 电化教育、电化教育研究、现代教育技术、中国远程教育、现代远距离教育、开放教育研究、 外语电化教学) 作为本研究的文献来源。在 CNKI（中国期刊全文数据库）上, 以 “个性化学 习” 为主题, 以 “远程教育杂志, 中国电化教育, 电化教育研究, 现代教育技术, 中国远程教育, 现代远距离教育, 开放教育研究, 外语电化教学” 为文献来源, 截止 2016 年 5 月 21 日共搜索 到文献 280 篇，去除会议通知等不相关文献后剩余 277 篇有效文献。

2.数据处理

对 277 篇有效文献进行高频关键词的确定，依次来确定数据的相似矩阵和相异矩阵，从 而对关键词进行共词聚类分析和社会网络分析。我们依据普莱斯计算公式来确定高频关键词。 即 $M=0.749 \sqrt{N_{\text {max }}}$ ，其中 $M$ 代表高频阈值， $N_{\text {max }}$ 代表一定时间范围内论文被引频次的最高 数值 ${ }^{[10]}$ 。搜索发现, 被引频次最高的文献是杨玉芹的 “MOOC 学习者个性化学习模型建构”, 被引用率为 41 。由普莱斯公式得出高频國值 $M$ 为 5 。由此我们选取频次 $\geq 5$ 的关键词作为高 频关键词, 共 33 个, 占所有 742 个关键词的 4. 45\%。然后利用文献题录信息统计分析工具 SAYI3. 2 创建高频关键词共现矩阵和相异矩阵, 如表 1 和表 2 所示。

表 1 高频关键词共现矩阵 (部分)

\begin{tabular}{l|c|c|c|c|c}
\hline & 个性化学习 & E-learning & 学习分析 & 移动学习 & 翻转课堂 \\
\hline 个性化学习 & 67 & 4 & 5 & 2 & 3 \\
\hline E-1earning & 4 & 14 & 1 & 0 & 0 \\
\hline 学习分析 & 5 & 1 & 13 & 0 & 0 \\
\hline 移动学习 & 2 & 0 & 0 & 13 & 1 \\
\hline 翻转课堂 & 3 & 0 & 0 & 1 & 13 \\
\hline
\end{tabular}

表 2 高频关键词相异矩阵（部分）

\begin{tabular}{l|c|c|c|c|c}
\hline & 个性化学习 & E-1earning & 学习分析 & 移动学习 & 翻转课堂 \\
\hline 个性化学习 & 0 & 0.9829 & 0.9713 & 0.9954 & 0.9897 \\
\hline E-1earning & 0.9829 & 0 & 0.9945 & 1 & 1 \\
\hline 学习分析 & 0.9713 & 0.9945 & 0 & 1 & 1 \\
\hline 移动学习 & 0.9954 & 1 & 1 & 0 & 0.9941 \\
\hline 翻转课堂 & 0.9897 & 1 & 1 & 0.9941 & 0 \\
\hline
\end{tabular}

\section{3. 数据统计与分析}

\section{1 我国个性化学习研究主题的基本类别}

本研究选择系统聚类的方法将高频关键词相异矩阵（即表 2) 进行聚类分析。具体做法 为：依次选择 “分析一分类一系统聚类” ，将聚类方法选定为 “组间联结”， “度量标准一 区间” 选定为 “Pearson 相关性” , 以 “变量” 为分群指标, 以 “树状图” 为分析结果呈现 形式, 结果如图 1 所示: 


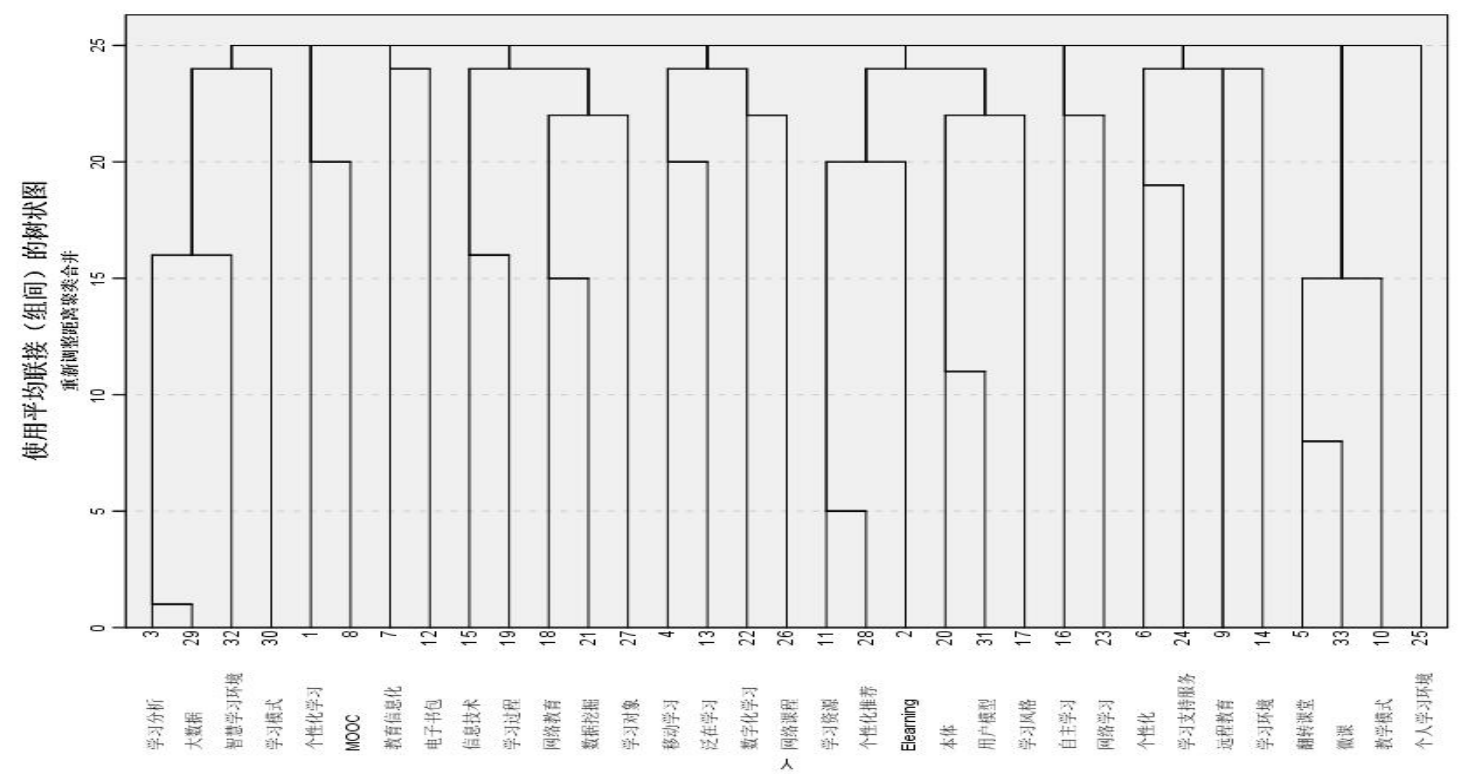

图 1 高频关键词聚类树状图

通过观察分析高频关键词聚类树状图, 可将个性化学习研究主题领域大致分为: 个性化 学习技术支撑, 主要包含学习分析、大数据、数据挖掘等相关技术; 个性化学习理论支撑, 主要包括学习风格的分析与测量、用户模型构建、个性化学习策略、个性化学习需求以及个 性化学习模式等相关内容。此外, 结合对个性化学习的认识和理解, 增加个性化学习基础理 论和个性化学习评价两个类别。

1. 个性化学习基础理论

个性化学习的理论渊源最早可追溯到我国教育家孔子 “因材施教” 的教学思想。他认为, 教学要根据每个学生不同的学习特点进行个别化教学, 强调学习者之间学习方式、学习需求 的差异性、独特性。

杨南昌学者于 2003 年提出多元智能理论指导下个性化学习环境设计的相关策略 ${ }^{[11]}$ 。文中 指出, 多元智能理论强调每个个体拥有多种不同的智力。不仅不同个体之间的智力发展有所 差异, 且同一个体所拥有的智力水平也会表现出不同。由此, 个体所拥有的智力结构及其所 表现出来的发展水平差异就构成了每个人的个别化、差异化学习特点。教学就应当根据学生 不同的智力水平予以适合的学习资源及路径支持。

郑云翔学者则从多元智能理论、分布式认知理论、人本主义学习理论以及新建构主义学 习理论的角度探讨了个性化学习的主要特征。他认为个性化学习要尊重学习者的个体差异, 不同的学习者在学习风格、学习需要、认知水平等方面都会表现出差异性和个别化, 应当根 据学生的不同特点给予个性化的学习服务和支持; 个性化学习要注重学生的个性发展, 强调 学生个性特征的自由成长, 应以知识创新为最终目标, 促使学生以自我认知为基础的意义建 构; 个性化学习强调给予学习者更多表达和交流的机会, 让学生在互动中相互成长、互享心 得体会, 从而促使自我的和谐发展; 个性化学习需要借助个性化技术为学生提供个性化的学 习支持，以实现学生的个性化成长 ${ }^{[12]}$ 。

我国学者对个性化学习基础理论的探讨不是很多, 但都强调了多元智力理论对个性化学 习的支持和发展。这说明, 个性化学习强调学生拥有多重智力, 且学生之间的智力有所差异。 由此, 教学就应当关注学生个体之间不同的智力倾向和学习差异, 进行个别化、差异化的教 学和辅导。

2. 个性化学习技术支撑

个性化学习的实现离不开技术的支撑与服务。个性化学习资源推送、个性化学习路径选 择以及个性化学习过程实时监控与评价等都需要多样化、多渠道的资料来源和智能化学习工 
具的支持。近年来, 学习分析技术、大数据、数据挖掘以及可视化等技术在教育领域的应用 和发展, 也推动着学生个性化学习的促成与实现。

纵观我国学者关于个性化学习技术支撑方面的研究, 主要集中在个性化学习系统/平台设

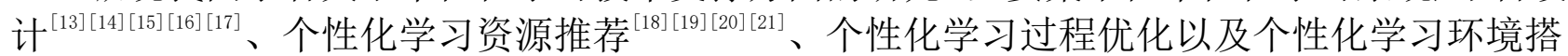
建，涉及到的技术有学习分析、DVR 技术、协同过滤技术、超媒体技术、Agent 技术以及静脉 感知技术等。其中, 学习分析主要通过对数据进行收集、分析, 建立实时监控、动态反馈的 个性化学习平台, 从而实现学生的个性化学习支撑和服务 ${ }^{[22]}$ 。协同过滤技术主要通过收集学 生信息, 建立学生的个性化学习档案, 进而根据学生档案进行个性化学习行为的分析和预测, 以此提供优化的个性化教育资源和及时的个性化学习评估 ${ }^{[23]}$ 。此外, 智慧学习理论 ${ }^{[24]}$ 、电子 书包 ${ }^{[25]}$ 等教育教学新思路、新工具也为个性化学习的实现与发展提供了新的视角。

由此可见, 我国学者关于个性化学习技术支撑方面的研究颇多, 涉及到的技术广泛, 在 个性化学习实现中应用的角度也比较丰富。但究其结果而言, 目前技术支持下的个性化学习 实现并非十分理想, 一方面由于技术应用于教育, 尤其是个性化学习, 需要一定的适应和衔 接; 另一方面, 目前对于个性化学习的实现路径、资源推送策略及个性化指导的理论研究并 不丰富。技术是实现个性化学习的保证, 而理论是实现个性化学习的基础。

3. 个性化学习理论支撑

个性化学习理论支撑主要涉及从个性化学习策略设计、个性化学习风格分析到个性化学 习模型构建、个性化学习环境设计等几方面内容。

个性化学习策略设计主要包括个性化学习资源推送策略和个性化学习服务策略等。对于 个性化学习资源推送策略而言, 孙众学者从教师、学生和系统三个角度分析并提出数字教材 中个性化学习资源的推送策略 ${ }^{[26]}$; 李宝学者则从学习者特征模型构建的角度, 通过将其与资 源特征进行相似度匹配, 进而来完成对学习资源推送服务模型的构建, 达到学习资源个性化 推送的目的 ${ }^{[27]}$ 。对于个性化学习服务策略而言, 李书明学者分别从个性化学习资源服务和个 性化学习过程服务两个角度进行了相关论述 ${ }^{[28]}$; 徐超学者则从人员角色的分配与管理角度构 建了 “三对一” 全程跟踪管理模式, 将教学管理人员、学科教师代表以及学生督学人员作为 学生进行个性化学习的监督导学体系人员构成, 以保障学生的个性化学习 ${ }^{[29]}$ 。

分析学生学习风格有助于更好的为学生提供个性化的支持和服务。王聪学者就学习风格 相关基础理论研究归纳总结出了学习风格的五种分析要素, 并依据此要素进行量表设计、实 证研究, 通过数据分析学习者学习风格各要素的特征属性 ${ }^{[30]}$; 杨娟学者则从 Felder-Silverman 学习风格模型中选取了 4 个关键维度构建了学习风格模型, 并分析了不同维度下学习风格对 资源组织所产生的影响 ${ }^{[31]}$ 。

个性化学习模型主要包括学生进行个性化学习的过程设计及其实现条件等因素。其中, 杨玉芹学者就 MOOC 相关学习理念和实践共同体等思想构建了 MOOC 学习者个性化学习模型, 模型包括过程及其支持条件（即环境、技术以及评价几个方面的相互依存和组合） ${ }^{[32]}$ 。韩建 华学者则依据个性化学习理论和学习分析等相关理论, 构建了智能导学系统下的个性化学习 过程模型, 并验证了其有效性 ${ }^{[33]}$ 。此外, 对于个性化学习模式而言, 目前学界并无对其确定 的概念界定和理论阐释, 现有研究主要聚集一定环境下的模式构建, 如信息技术环境 ${ }^{[34]}$ 、网 络环境 ${ }^{[35]}$ 以及 Web2.0 环境 ${ }^{[36]}$ 等。

个性化学习环境是学生开展个性化学习的基础土壤。目前关于环境构建的研究有熊玉珍 学者基于测评技术所构建的汉语学习个性化环境 ${ }^{[37]}$ 以及杨进中学者基于社交网络模型构建的 个性化学习环境, 并将其环境设计要素确定为个人学习空间、学习资源聚合模式、个性化推 荐引擎构建三个方面 ${ }^{[38]}$ 。

综观以上对于个性化学习支撑的理论研究, 可以认为, 对学生个性化学习风格的分析研 究是学习资源个性化推送、学习路径个性化支持和学习服务个性化提供的基础前提, 并在此 基础上, 多视角、多维度的剖析学习过程, 结合相关学习理论构建个性化学习模型、模式或 
环境, 从而达到学生个性化学习的目的。而目前个性化学习发展成效不大的原因, 可能存在 于个性化学习相关支持对学习过程中时时的学习需求关注不够, 以及对影响不同学生学习的 关键因素掌握不够。

4. 个性化学习评价

个性化学习评价在整个个性化学习活动中起着 “提供反馈指导、提高学习质量” 的作用。 在该方面的研究中, 牟智佳学者就大数据和电子书包的功能特点, 提出了内容评价上的个性 化学习评价模型和结果评价上的个性化评价层次塔理论, 为个性化学习的评价研究提供了新 的视角和思路 ${ }^{[39]}$; 陈敏学者则从学生学习过程中所产生的信息出发, 提出了基于过程性信息 的个性化学习评价方法, 评价维度涉及了学习态度、学习活动等多视角的过程性信息 ${ }^{[40]}$ 。

目前个性化学习领域对该方面的研究并不丰富, 缺乏系统化、多视角的理论观点和实践 研究。本文认为, 评价不仅应涉及到内容评价、结果评价, 还要关注过程评价以及学生学习 态度、学习风格的养成等软因素的评价。这些因素是学生能够长期稳定的进行高效学习的内 在保障。而对于个性化学习评价而言, 应当更加关注学生的自我评价以及在教师或同伴指导 交流下的自我评价。

\section{2 我国个性化学习研究主题的热点}

将高频关键词共现矩阵通过社会网络图谱分析软件 Ucinet6. 2 中的 Netdraw 工具进行可 视化分析, 可以得到如图 2 所示的高频关键词共现网络图谱。它能够揭示高频关键词之间的 相互关联关系，从而获悉所研究主题的热点情况。

观察图 2, 不难发现, 各高频关键词形成了以个性化学习为核心的网络图谱, 并呈现了 以数据挖掘、MOOC、学习环境、学习分析、泛在学习、学习风格、E-1earning 以及教学模式 等关键词为主的核心关键词区域。表明, 此类关键词与个性化学习的关联较紧密, 是个性化 学习研究领域中的热点主题。究其原因, 我们认为, 学生个性化学习需求的获取、学习风格 的分析以及个性化学习环境的创设是个性化学习实现的前提和保障。学习需求等信息的获取 能够为学生个性化学习资源支持和个性化学习路径引导提供数据支撑。学习风格的分析与判 定也是为学生提供个性化学习服务的重要策略依据。个性化学习环境的构建更是引导学生开 展个性化学习的基础土壤。由此, 以大数据、学习分析为核心的数据挖掘技术成为目前个性 化学习研究的主流技术。学习风格的分析模型与个性化学习环境的构建方法也得到了个性化 学习研究者的持续关注。

此外, 图谱中的其他主题, 如远程教育、网络课程、智慧学习环境、自主学习、网络学 习、个性化推荐、微课、学习支持服务等关键词处于相对边缘的位置。表明, 其与个性化学 习的关联较弱。关联较弱不代表相应的主题一定不是研究重点, 一方面可能是由于目前技术 或理论研究尚不能够推动该主题的发展, 另一方面也可能是由于目前的研究尚未关注到该主 题的重要性。

从网络图谱的整体视角而言，该图谱节点分布稀疏，无较明显的集中倾向。表明，个性 化学习相关主题研究较平稳、均衡, 并无较明显的热点主题。从网络图谱内核、外核区域高 频关键词的属性类别来看, 对个性化学习的数据分析、资源推荐等技术研究较多, 对其的实 现与应用情况较少。 


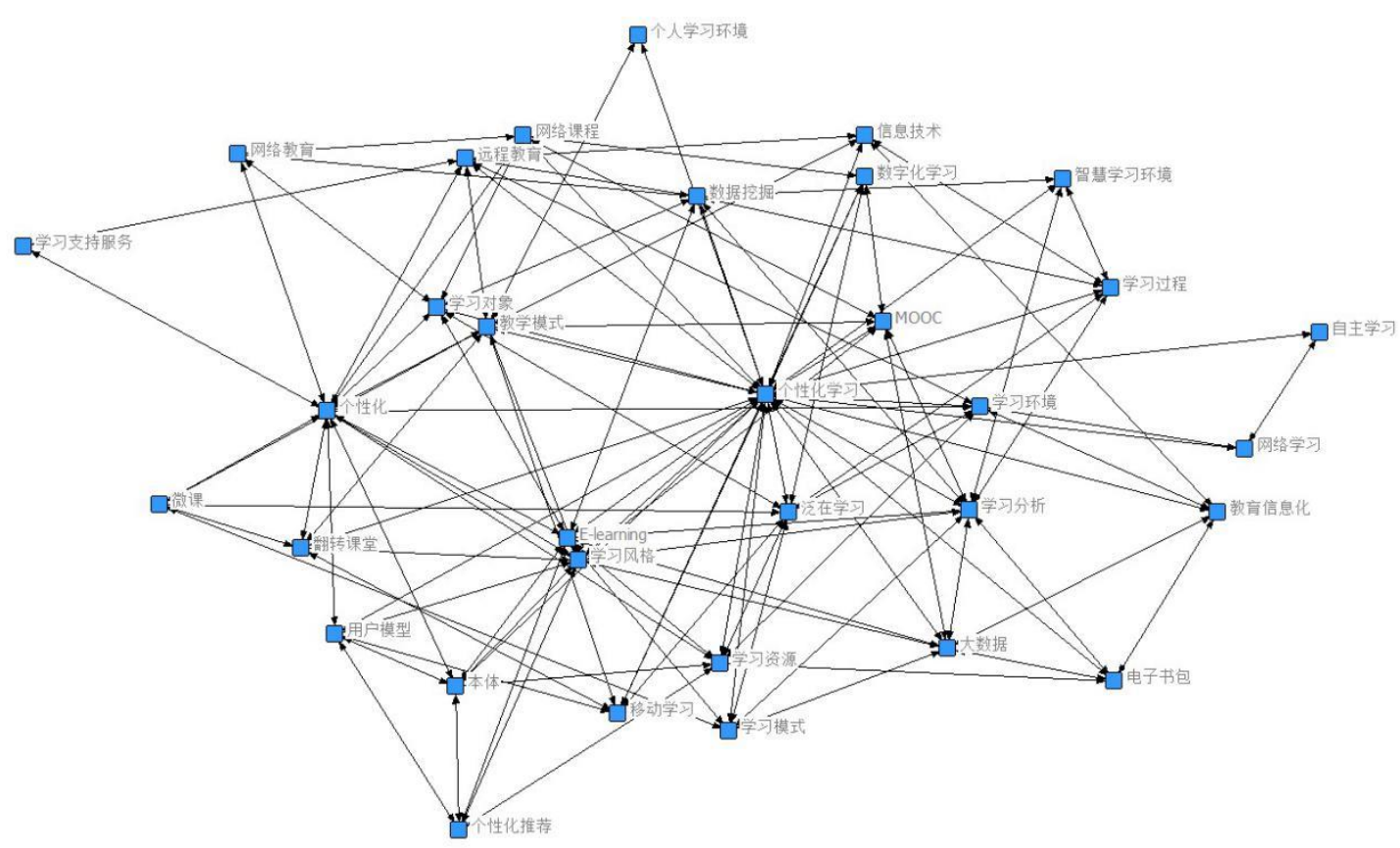

图 2 高频关键词共现网络图谱

\section{3 我国个性化学习研究主题的发展趋势}

对高频关键词共现矩阵利用 Ucinet6. 2 中的 Network-centrality-multiple measures 工 具进行计算, 得到如表 3 所示的结果。其中, 点度中心度表示该节点所代表的主题自身的交 互能力, 接近中心度表示节点所代表的主题不受其他主题影响的能力, 而中间中心度则表示 节点所代表的主题控制其他主题发展的能力 ${ }^{[41]}$ 。因此, 一般认为, 点度中心度和接近中心度 数值相对较高, 且中间中心度数值相对较低的主题能够在一定程度上反应所研究领域未来的 发展趋势 ${ }^{[42]}$ 。

表 3 高频关键词中心度统计结果（部分）

\begin{tabular}{|c|c|c|c|c|}
\hline & 关键词 & 点度中心度 & 接近中心度 & 中间中心度 \\
\hline 1 & 个性化学习 & 84.375 & 86.486 & 43.242 \\
\hline 2 & E-1earning & 31.250 & 59.259 & 3.435 \\
\hline 3 & 学习分析 & 31.250 & 58.182 & 2.329 \\
\hline 4 & 移动学习 & 18.750 & 53.333 & 0.630 \\
\hline 5 & 翻转课堂 & 18.750 & 55.172 & 0.630 \\
\hline 6 & 个性化 & 43.750 & 60.377 & 10.746 \\
\hline 7 & 教育信息化 & 15.625 & 50.794 & 0.437 \\
\hline 8 & M00C & 21.875 & 55.172 & 0.904 \\
\hline 9 & 远程教育 & 21.875 & 55.172 & 3.225 \\
\hline
\end{tabular}

从表 3 可以看出, 个性化、教学模式、 E-1earning、远程教育、学习风格、泛在学习、 学习资源、学习分析、数据挖掘、学习环境等关键词的点度中心度和接近中心度相对较低, 且其中间中心度相对较高。表明, 这些关键词能够在一定程度上反映我国个性化学习未来一 段时间的研究趋势。具体分为以下三个方面:

1. 个性化学习的理论架构方法

以个性化、教学模式、学习环境等关键词为代表的个性化学习理论知识架构方法在未来 一段时间内将成为该领域的研究热点。个性化的教学模式主要指在一定的教与学理论指导下 开展的能够为学生提供个性化学习服务的教学活动、学习资源、学习路径等一系列支持的教 学形式。个性化的学习环境不仅包括网络学习平台、学习空间、自适应学习系统等技术应用, 学生的外在学习环境（如教室布局、灯光亮度、座椅样式等）以及内在软环境（如学习氛围、 
学生之间的关系融洽度等）也会对学生个性化学习开展带来关键性的影响。

2. 个性化学习的多元化存在形式

以 E-1earning、远程教育、泛在学习等关键词为代表的个性化学习多元化存在形式也势 必会成为个性化学习研究的发展趋势。技术及其在教育领域的应用与发展, 促使多样化的在 线学习形式不断涌现。而个性化学习与其说是一种学习形式, 不如说是一种学习理念。独立 存在的个性化学习活动必然会引起脱离教学实际等问题, 而将其与其他多元化的在线学习形 式相结合符合目前技术促成多样化学习的趋势和发展。

3. 个性化学习的实现途径与技术

以学习风格、学习分析、数据挖掘、学习资源等关键词为代表的个性化学习实现途径与 技术是实现学生个性化学习的技术保障。只有了解学生的学习风格, 以其学习特点、学习倾 向等因素为依据, 才能准确的设计出符合学生学习规律的教学活动, 并为其提供适宜的学习 资源、学习引导等服务。而这些信息的获取也必然会将数据挖掘、学习分析等技术应用其中。 但目前该方面研究还存在诸多问题, 如学生学习相关数据如何获取、获取哪些信息以及获取 到的信息如何利用等，这将是个性化学习研究中下一步的工作重点。

\section{4. 总结与展望}

凡事都有一个循序渐进、不断发展的过程, 个性化学习的实现与发展也不可能一䟣而就、 一气呵成。个性化学习虽由来已久, 但其基础理论、技术应用以及效果评价等方面的研究发 展并不成熟, 还需进一步的探讨与实践。个性化学习的实现意义不仅在于为学生提供个性化 的学习服务、提高学生学习效率, 更在于促使学生发现自己的学习规律、学习特点, 以形成 自主学习、终身学习的学习理念。这将是个性化学习乃至整个教育的价值趋向。

\section{致谢}

本文为国家科技支撑计划 “区域互动教研与培训学分交互应用示范”（课题批准号: 2014BAH22F05）的阶段性成果之一。

\section{References}

[1]Dewei HU, Education theory of individualized learning and its importance to education from New Zealand minister, Basic Education Review, vol.10, pp. 28-30, 2006.

[2] The central people's government of the People's Republic of China. The national medium and long-term education reform and development plan outline (2010-2020), [EB/OL].2010. http://www.gov.cn/jrzg/2010-07/29/content_1667143.htm,2014-02-12.

[3]Jinping $\mathrm{Xi}$ to international conference on education informationization of congratulations[EB/OL].2015.http://news.xinhuanet.com/politics/2015-05/23/c_1115383959.ht $m, 2015-05-22$.

[4] Weijing Zhong, Jia Li, The Research of Co- word Analysis(1)— the Process and Methods of Co- word Analysis, Journal of Information,vol.05,pp.70-72,2008.

[5] Weijng Zhong,Jia Li,Xingju Yang. The Research of Co- word Analysis(3)— The Principle and Characteristics of the Co- Word Cluster Analysis, Journal of Information,vol.07,pp.118-120,2008.

[6] Qinghua Zhu,Liang Li,Social network analysis method and its application in information science, Information Studies:Theory \& Application, vol.02,pp.179-183+174,2008.

[7]Ming Xiao,The use guide of Knowledge map tools, China Railway Publishing 
House,pp.37-38,2014.

[8] Yunjing Li,Research of knowledge map building based on citation analysis visualization, Southeast university press,pp.11-12,2009.

[9] Xin Jiang, The application research of Social network analysis method in the field of book intelligence, Intellectual property rights press,pp.123-126,2015.

[10] Youmei wang, Haiyan Wu,Analysis of Academic Characteristic of the Highly Cited Papers in Chinese Higher Education Field_—Based on the bibliometrics of Literatures from China Higher Education Research (2000-2011), China Higher Education Research,vol.01,pp.33-37,2012.

[11] Nanchang Yang, Zhixian Zhong, Enlightenment of The multiple intelligences theory to personalized network learning environment design, Distance Education in China, vol.03,pp.48-50,2003.

[12]Yunxiang Zheng, Research on Teaching Models for College Students' Personalized Learning from the Perspective of New Constructivism, Journal of Distance Education, vol.04,pp.48-58,2015.

[13]Yanwen $\mathrm{Wu}, \mathrm{Zhenghong} \mathrm{Wu}$, Design of intelligent question-answering platform based on the learners' personality model, e-Education Research,vol.06,pp.64-66+70,2005.

[14]Hui Deng, Analysis and design of the network personalized learning students characteristics analysis system, Journal of Distance Education, vol.01,pp.11-13,2003.

[15]Yanbai Jia,Xiaojun Zhou,Shuangqing Qian, Zhigang Yang, Lingping Gong, The construction and application of personalized intelligent learning service system, Distance Education in China, vol.10,pp.71-74,2007.

[16] Xin Li,Constructing distributed virtual learning environment based on DVR technology, China Educational Technology, vol.01,pp.69-73,2011.

[17] Xinlai Dai,Nannan Zhang,Jian Wang,Construction and technical implementation of personalized education resources platform master of education oriented, e-Education Research,vol.01,pp.69-73,2011.

[18]Lina Yang,Kecheng Liu,Zhijun Yan,Learning resources personalized recommendation research oriented to virtual learning community, e-Education Research,vol. 04,pp. 67-71+85,2010.

[19] Qiang Jiang,Wei Zhao,Xin Du,Ming Liang,Research about personalized ontology learning resources recommended research based on the user model, China Educational Technology, vol.05,pp.106-111,2010.

[20]Wei Zhao,Qiang Jiang,Pengjiao Wang,Liping Wang, Ontology-driven Personalized Recommendation of Knowledge Resource in e-Learning, China Educational Technology, vol.05,pp.84-89,2015.

[21]Lina Yang,Kecheng Liu,Zhijun Yan,Research on the personalized learning resources recommended under the framework of case-based reasoning Agent cooperation, China Educational Technology, vol.12,pp.105-109,2009.

[22] Xiaoming Cao, Yong Zhu,Research on personalized learning platform under the perspective of learning analysis, Open Education Research, vol.05,pp.67-74,2014.

[23] Yonggu Wang,Feiyue Qiu,Jianlong Zhao,Hui Liu,Research on Personalized Recommendation of Learning Resources Based on Collaborative Filtering Recommendation Technology, Journal 
of Distance Education,vol.03,pp.66-71,2011.

[24] Hongyan Wu,Research on Personalized Learning System under the Perspective of Smart Learning, China Educational Technology, vol.06,pp.127-131,2015.

[25] [39]Zhijia Mou,Research on Model of Personalized Learning Evaluation System Based on Educational BigData in the Electronic Schoolbag, Journal of Distance Education,vol.05,pp.90-96,2014.

[26]Zhong Sun,Liming Luo,Xin Qi, The push strategy and the technical implementation of personalized learning resources about Digital textbooks, e-Education Research,vol.09,pp.64-70,2014.

[27]Bao Li, Wenlan Zhang, The Construction of Learning Resources Push Service Model under the Smart Educational Environment, Journal of Distance Education,vol.03,pp.41-48,2015.

[28] Shuming Li,Jun Tian,Research about strategy on personalized learning services in network learning, China Educational Technology, vol.06,pp.118-121,2011.

[29]Chao Xu,Qiang Li,Yongxu Ma, Personalized learning support services for the disabled mode of practice and exploration, Distance Education in China, vol.09,pp.52-55,2012.

[30] Cong Wang,Dong Wang,A Study on Learners' Learning Styles of College English in Adaptive Learning Systems Environment, China Educational Technology, vol.07,pp.145-149,2014.

[31] Juan Yang,Yangli Zhang,Zhixing Wang,Hongtao Liu,Xinglu Huang,Smap: adaptive Felder-Silverman learning style model of dynamic learning path recommendation tool, Distance Education in China, vol.05,pp.77-86,2013.

[32] Yuqin Yang,Three Emerging Models of Micro Curriculum Development, China Educational Technology, vol.06,pp.6-10+68,2014.

[33] Jianhua Han,Qiang Jiang,Wei Zhao,Dongliang Liu,A Model of Personalized Learning in Intelligent Tutoring Environment and Its Evaluation, e-Education Research,vol. 07,pp. 66-73,2016.

[34] Yunxiang Zheng,goal-driven personalized learning model under the information technology support, e-Education Research,vol. 07,pp. 89-92,2010.

[35] Zhihui Jiang,A Study of Measuring Model for Information Resources of China Higher

Education Based on Affluent Coefficient of Information Resources, Distance Education in China, vol.02,pp.48-51+95,2013.

[36] Zhiyong Zhong,Construction and application of personalized learning model based on Web2.0 environment, China Educational Technology, vol.08,pp.107-110,2012.

[37]Yuzhen Xiong, Construction of personalized learning environment of Chinese based on the evaluation, e-Education Research,vol. 03,pp. 69-7+87,2012.

[38] Jinzhong Yang,Jianping Zhang,Research on construction of personalized learning environments based on social network, Open Education Research,vol.02,pp.89-97,205.

[40] Min Chen,Xianmin Yang,How to Promote Online Dialogue and Interaction among Teachers and University, China Educational Technology, vol.06,pp.21-26+120,2016.

[41] Lu Wang,Social network structure of the virtual learning community, Peking University Press,pp.95-102,2011.

[42] Haifeng Li,Wei Wang,Study on Hot Research Topics on International MOOC and Its 
Development Trend:Knowledge Visualization Mapping Analysis Based on Co-word Analysis Method, Journal of Distance Education, vol.05,pp.38-45,2015. 